\title{
The Large Scale Distribution of Galaxies in the Shapley Supercluster
}

\author{
Michael J. Drinkwater, ${ }^{1}$ Quentin A. Parker, ${ }^{2,3}$ Dominique Proust, ${ }^{4}$ Eric Slezak, ${ }^{5}$ \\ and Hernán Quintana ${ }^{6}$ \\ ${ }^{1}$ Department of Physics, University of Queensland, QLD 4072, Australia \\ (e-mail: mjd@physics.uq.edu.au) \\ ${ }^{2}$ Department of Physics, Macquarie University, NSW 2109, Australia \\ ${ }^{3}$ Anglo-Australian Observatory, PO Box 296, Epping, NSW 1710, Australia \\ (e-mail: qap@ics.mq.edu.au) \\ ${ }^{4}$ GEPI - Observatoire de Paris-Meudon, 92195 Meudon CEDEX, France \\ (e-mail: Dominique.Proust@obspm.fr) \\ 5 Observatoire de Nice, 06304 Nice CEDEX4, France \\ (e-mail: Slezak@obs-nice.fr) \\ ${ }^{6}$ Departamento de Astronomia y Astrofisica, Pontificia Universidad Católica de Chile, \\ Casilla 104, Santiago 22, Chile \\ (e-mail: hquintan@astro.puc.cl)
}

Received 2003 October 13, accepted 2003 December 10

\begin{abstract}
We present new results of our wide-field redshift survey of galaxies in a 182 square degree region of the Shapley Supercluster (SSC) based on observations with the FLAIR-II spectrograph on the UK Schmidt Telescope (UKST). In this paper we present new measurements to give a total sample of redshifts for 710 bright $(R \leq 16.6)$ galaxies, of which 464 are members of the SSC $\left(8000<v<18000 \mathrm{~km} \mathrm{~s}^{-1}\right)$. Our data reveal that the main plane of the SSC $\left(v \approx 14500 \mathrm{~km} \mathrm{~s}^{-1}\right)$ extends further than previously realised, filling the whole extent of our survey region of 10 degrees by 20 degrees on the sky (35 Mpc by $70 \mathrm{Mpc}$, for $\left.H_{0}=75 \mathrm{~km} \mathrm{~s}^{-1} \mathrm{Mpc}^{-1}\right)$. There is also a significant structure associated with the slightly nearer Abell 3571 cluster complex $\left(v \approx 12000 \mathrm{~km} \mathrm{~s}^{-1}\right)$ with a caustic structure evident out to a radius of $6 \mathrm{Mpc}$. These galaxies seem to link two previously identified sheets of galaxies and establish a connection with a third one at $\bar{V}=15000 \mathrm{~km} \mathrm{~s}^{-1}$ near RA $=13^{\mathrm{h}}$. They also tend to fill the gap of galaxies between the foreground HydraCentaurus region and the more distant SSC. We calculate galaxy overdensities of $5.0 \pm 0.1$ over the 182 square degree region surveyed and $3.3 \pm 0.1$ in a 159 square degree region excluding rich clusters. Over the large region of our survey the inter-cluster galaxies make up 46 per cent of all galaxies in the SSC region and may contribute a similar amount of mass to the cluster galaxies.
\end{abstract}

Keywords: Redshifts of galaxies — clusters of galaxies — subclustering

\section{Introduction}

In the past few decades, large galaxy redshift surveys have revealed structures on ever-increasing scales. The largest structures found are superclusters, collections of thousands of galaxies with sizes as large as $100 \mathrm{Mpc}$. The mere existence of these structures places important constraints on theories of the formation of galaxies and clusters. The Shapley supercluster (SSC), the subject of this paper, is one of the most massive concentrations of galaxies in the local universe (Scaramella et al. 1989; Raychaudhury 1989), so it is also of particular interest to consider its effect on the dynamics of the Local Group.

The SSC is a remarkably rich concentration of galaxies centred around $\mathrm{RA}=13^{\mathrm{h}} 25^{\mathrm{m}}$, Dec $=-30^{\circ}$ which has been investigated by numerous authors since its discovery in 1930 (see Quintana et al. 1995). It consists of many clusters and groups of galaxies in the redshift range $0.04<z<0.055$. The SSC lies in the general direction of the dipole anisotropy of the Cosmic Microwave Background (CMB) (Smoot et al. 1992), and is located $130 \mathrm{~h}^{-1} \mathrm{Mpc}$ beyond the Hydra-Centaurus supercluster (itself about $50 \mathrm{~h}^{-1} \mathrm{Mpc}$ away from us). Quintana et al. (1995) estimated that for $\Omega_{0}=0.3$ and $H_{0}=75 \mathrm{~km} \mathrm{~s}^{-1} \mathrm{Mpc}^{-1}$, the gravitational pull of the supercluster may account for up to $25 \%$ of the peculiar velocity of the Local Group required to explain the CMB dipole anisotropy, in which case the mass of the supercluster would be dominated by inter-cluster dark matter. A major study of the SSC was made by Bardelli et al. (2000, 2001, and references therein) who also studied inter-cluster galaxies in the core region of the supercluster. They derived a total mean overdensity of the SSC of $N / \bar{N} \approx 11.3$ on a scale of $10.1 \mathrm{~h}^{-1} \mathrm{Mpc}$ and found 
that the central part of the supercluster contributes about $26 \mathrm{~km} \mathrm{~s}^{-1}$ to the peculiar velocity of the Local Group.

The early studies of the SSC were limited (primarily by observational constraints) to the various rich Abell galaxy clusters in the region, but this might give a very biased view of the overall supercluster as the clusters represent only the most concentrated peaks in the luminous matter distribution. We have commenced an investigation into the larger scale distributions of galaxies throughout the entire SSC region and close environs using data from wide-field multi-fibre spectrographs such as the FLAIR-II system on the UK Schmidt Telescope (UKST) of the AngloAustralian Observatory (Parker \& Watson 1995; Parker 1997). With such multiplexed facilities we are able to measure many more galaxy redshifts over large angular extents and obtain a more complete picture of the composition and disposition of galaxies in the entire supercluster.

A preliminary analysis based on 301 new, bright $(R \leq 16)$ galaxy redshifts obtained with FLAIR-II in a $77 \mathrm{deg}^{2}$ region of the supercluster has already been published by our group (Drinkwater et al. 1999, hereafter D99). The measured galaxies were uniformly distributed over the selected inter-cluster regions, and most ( $230 \simeq 75 \%)$ were found to be members of the supercluster. The D99 sample traced out two previously unknown sheets of galaxies which appeared to link various Abell clusters in the supercluster. We also found that in a $44 \mathrm{deg}^{2}$ sub-area of the supercluster excluding the Abell clusters, these sheets alone represent an overdensity of a factor of $2.0 \pm 0.2$ compared to a uniform galaxy distribution. Within the initial survey area of D99, the new galaxies contribute an additional 50 per cent to the known contents of the SSC in that region, implying a corresponding increase in contribution to the motion of the Local Group. A much larger galaxy sample over a similar area of sky was presented by Quintana, Carrasco \& Reisenegger (2000). They measured nearly 3000 galaxy redshifts in an area of about $12 \mathrm{deg}$ by $6 \mathrm{deg}$. They estimated the upper bound on the mass of the central region and found the overdensity to be substantial, but still only able to contribute to a small fraction $\left(3 \Omega_{m}^{-0.4} \%\right)$ of the observed motion of the Local Group (Reisenegger et al. 2000).

In this paper we present radial velocities for an additional 409 bright $(R \leq 16.5)$ galaxies spread over an extended region to the East, West and South of the main SSC concentration. We analyse a combined magnitudelimited sample from this paper and D99 of 710 galaxies with measured redshifts, of which 464 (65\%) are members of the supercluster based on their redshift range $\left(8000<c z<18000 \mathrm{~km} \mathrm{~s}^{-1}\right)$. They seem to link the two previously identified galaxy sheets found in D99 and also establish a connection with a third feature at $\bar{V}=15000 \mathrm{~km} \mathrm{~s}^{-1}$ near $\mathrm{RA}=13^{\mathrm{h}}$. The new sample also tends to fill a previous gap in the galaxy distribution between the foreground Hydra-Centaurus region and the SSC. Future study of the SSC will cover even larger regions using data from the FLASH survey of Kaldare et al. (2003) (also with the UKST/FLAIR-II system) and the $6 \mathrm{dF}$ survey of the southern sky currently in progress (Watson et al. 2000).

The observed galaxy sample and observations are described in Section 2, the results are presented in Section 3 and a brief discussion of the significance of these new measurements is given in Section 4. A full and detailed analysis and interpretation of the new redshifts from D99, this work and a compilation of all literature redshifts in the SSC is the subject of a separate paper (Proust et al., in preparation). Unless otherwise noted in this paper we use a Hubble constant of $H_{0}=75 \mathrm{~km} \mathrm{~s}^{-1} \mathrm{Mpc}^{-1}$, giving a distance of $200 \mathrm{Mpc}$ to the main supercluster $\left(c z=15000 \mathrm{~km} \mathrm{~s}^{-1}\right)$ so 1 degree subtends a distance of 3.5 Mpc.

\section{Observations and Data Reductions}

In 1997 we began a concerted campaign to obtain complete samples of galaxies down to the same magnitude below $L_{*}$ for constituent clusters and inter-cluster regions of the SSC using the FLAIR-II system at the UKST. During the period 1997-2000 we subsequently observed more than 700 selected galaxies over 7 UKST standard fields in the SSC region, namely in fields 382-384 and 443-446. The main SSC core centred on A3558 at $13^{\mathrm{h}} 25^{\mathrm{m}},-31^{\circ}$ is in field 444. As each selected Abell cluster has a projected angular diameter of 2.5 to 3.0 degrees, the wide-field UKST FLAIR-II system was an ideal facility for this project whilst additionally permitting us to probe the regions between the dominant clusters neglected in previous studies. These combined fields covered an area of $182 \mathrm{deg}^{2}$ which allows us to investigate the outer limits of the SSC out to radii of more than $10 \mathrm{deg}(35 \mathrm{Mpc})$ from $\mathrm{A} 3558$.

\subsection{Input Galaxy Samples}

All target galaxies were originally obtained from the red ESO/SRC sky survey plates of the above fields scanned by the Paris Observatory MAMA plate-measuring machine (Guibert \& Moreau 1991; Moreau 1992). The images were classified using standard star/galaxy separation techniques (e.g. Dickey et al. 1987; Heydon-Dumbleton, Collins \& MacGillivray 1989). We defined a galaxy sample to a photometric limit of $R \leq 16$, corresponding (assuming a mean $B-R=1.5$ ) to $B<17.5$, the nominal galaxy limiting magnitude of the FLAIR-II system (Parker \& Watson 1995). This corresponds to an absolute magnitude of $M_{B}=-19$ at the Shapley distance of $200 \mathrm{Mpc}$. This selection gave total galaxy samples of 600-1000 per field. All previously catalogued matches to literature redshifts were then removed from the target samples prior to observation.

\subsection{FLAIR-II Observations}

Table 1 gives the journal of FLAIR-II observations reported here. An interim magnetic-button fibre-ferrule system, implemented with the 71-fibre FLAIR-II plateholder, was commissioned during these observations. This 
Table 1. Summary of FLAIR-II Observations

\begin{tabular}{lcccclcr}
\hline Date & Field & RA $(J 2000)$ Dec & Time & Seeing & Weather & $\mathrm{N}_{g}$ & $\mathrm{~N}_{r}$ \\
\hline 1998 May 22 & F383B & $13: 15: 00-35: 15: 00$ & 5400 & 1 & Clear & 58 & 43 \\
1999 June 10 & F445A & $13: 51: 00-30: 15: 00$ & 11000 & $2-3$ & Clear & 65 & 53 \\
1999 June 11 & F445B & $13: 51: 00-30: 15: 00$ & 12000 & $3-4$ & Cloudy & 65 & 54 \\
1999 June 12 & F445C & $13: 51: 00-30: 15: 00$ & 12000 & 3 & Clear & 65 & 52 \\
1999 June 13 & F445D & $13: 51: 00-30: 15: 00$ & 12000 & $2-3$ & Clear & 65 & 41 \\
1999 June 14 & F443A & $13: 05: 00-30: 15: 00$ & 12000 & $1-2$ & Clear & 73 & 21 \\
1999 June 16 & F443B & $13: 05: 00-30: 16: 00$ & 12000 & $1-2$ & Clear & 73 & 32 \\
1999 June 17 & F443C & $13: 05: 00-30: 16: 00$ & 12000 & $2+$ & Cloudy & 65 & 42 \\
2000 June 2 & F446 & $14: 14: 00-30: 14: 00$ & 15000 & $2-3$ & Clear & 61 & 28 \\
2000 June 3 & F384 & $14: 03: 00-30: 14: 00$ & 15000 & $1-2$ & Clear & 80 & 64 \\
\hline
\end{tabular}

Notes: $\mathrm{N}_{g}$ is the number of target galaxies observed while $\mathrm{N}_{r}$ is the number of galaxies actually yielding a redshift in a particular field configuration, not counting the spectra dominated by galactic stars.

Table 2. Galaxies with Incorrect Measurements in D99

\begin{tabular}{lccl}
\hline $\begin{array}{l}\text { Original Position } \\
\text { RA (B1950) Dec }\end{array}$ & $\begin{array}{c}\text { New Position } \\
\text { RA (J2000) Dec }\end{array}$ & $\begin{array}{c}c z \pm \Delta c z \\
\left(\mathrm{~km} \mathrm{~s}^{-1}\right)\end{array}$ & Correction \\
\hline 13:04:08.4-37:06:37 & $13: 06: 56.1-37: 22: 09$ & - & Position \\
13:12:05.7-33:29:36 & $13: 14: 52.8-32: 45: 27$ & - & Position \\
13:14:37.9-33:39:08 & $13: 17: 26.2-33: 55: 36$ & - & Position \\
13:15:21.3-31:45:59 & $13: 18: 08.7-32: 01: 06$ & - & Position \\
13:18:06.6-27:47:20 & $13: 20: 52.8-28: 13: 06$ & - & Position \\
13:19:39.1-33:06:29 & $13: 22: 28.0-33: 29: 03$ & - & Position \\
13:24:21.4-37:00:34 & $13: 27: 13.1-36: 25: 34$ & - & Position \\
13:24:28.2-36:49:27 & $13: 27: 10.4-37: 05: 00$ & - & Position \\
13:29:40.1-33:54:18 & $13: 32: 31.3-33: 09: 42$ & - & Position \\
13:31:39.7-36:33:12 & $13: 34: 33.1-36: 48: 32$ & 239817 & Velocity \\
13:33:21.5-36:38:27 & $13: 36: 15.3-36: 53: 45$ & 389779 & Velocity \\
13:35:47.0-34:17:07 & - & - & Removed \\
13:37:15.1-34:05:41 & $13: 30: 06.0-34: 21: 10$ & - & Position \\
13:40:52.6-34:49:27 & $13: 43: 46.5-35: 04: 30$ & 1468171 & Velocity \\
13:42:33.6-36:31:58 & $13: 45: 29.1-36: 46: 59$ & 763380 & Velocity \\
13:43:08.2-33:29:39 & $13: 46: 01.4-33: 44: 39$ & 2483861 & Velocity \\
13:43:32.6-35:50:50 & - & - & Removed \\
\hline
\end{tabular}

Notes: ${ }^{1}$ the position given in $\mathrm{D} 99 ;{ }^{2}$ the $\mathrm{J} 2000$ position in this paper, corrected in some cases; ${ }^{3}$ in two cases the original position was wrong, but the correct identification could not be determined.

was as a proof of concept for the recently-commissioned automated robotic fibre positioner that will replace FLAIR-II at the UKST and known as 6dF (6-degree field) (e.g. Watson et al. 2000). Note that typically 6 fibres were devoted to the blank sky regions to facilitate skybackground subtraction. An overall redshift success rate of $75 \%$ was achieved from the FLAIR-II data.

The data were reduced as in Drinkwater et al. (1996) using the dofibers package in IRAF (Tody 1993). Redshifts were measured for galaxy spectra using the crosscorrelation task XCSAO in RVSAO (Kurtz \& Mink 1998) using a mixture of a dozen stellar and galaxy templates. The galaxy templates included a number of emission line examples. The IRAF EMSAO utility was also used to manually check emission line redshifts where necessary. For spectra showing both absorption and emission features, the result (either XCSAO/EMSAO) with the lower error was used. Note that 61 spectra were contaminated by a dominant foreground star although we tried to remove all galaxies with a dominant star on the line-of-sight during the fibering procedure. No redshift was measured for these galaxies.

The complete set of FLAIR-II measurements from both papers was carefully checked for consistency, in particular by matching the galaxy positions to the new SuperCOSMOS catalogue (Hambly et al. 2001a, 2001b, 2001c). During this process several errors in the data from D99 were identified: 5 galaxies had incorrect redshifts reported and 12 had incorrect positions, all but two of which we have been able to correct. The 17 galaxies from D99 with incorrect measurements are listed in Table 2.

Our complete catalogue of 710 galaxies measured in the work reported here and our previous FLAIR-II observations from D99 is reported in the Accessory Materials. The column descriptors are given at the end of the table. In Fig. 1 we show the projected distribution of the observed 


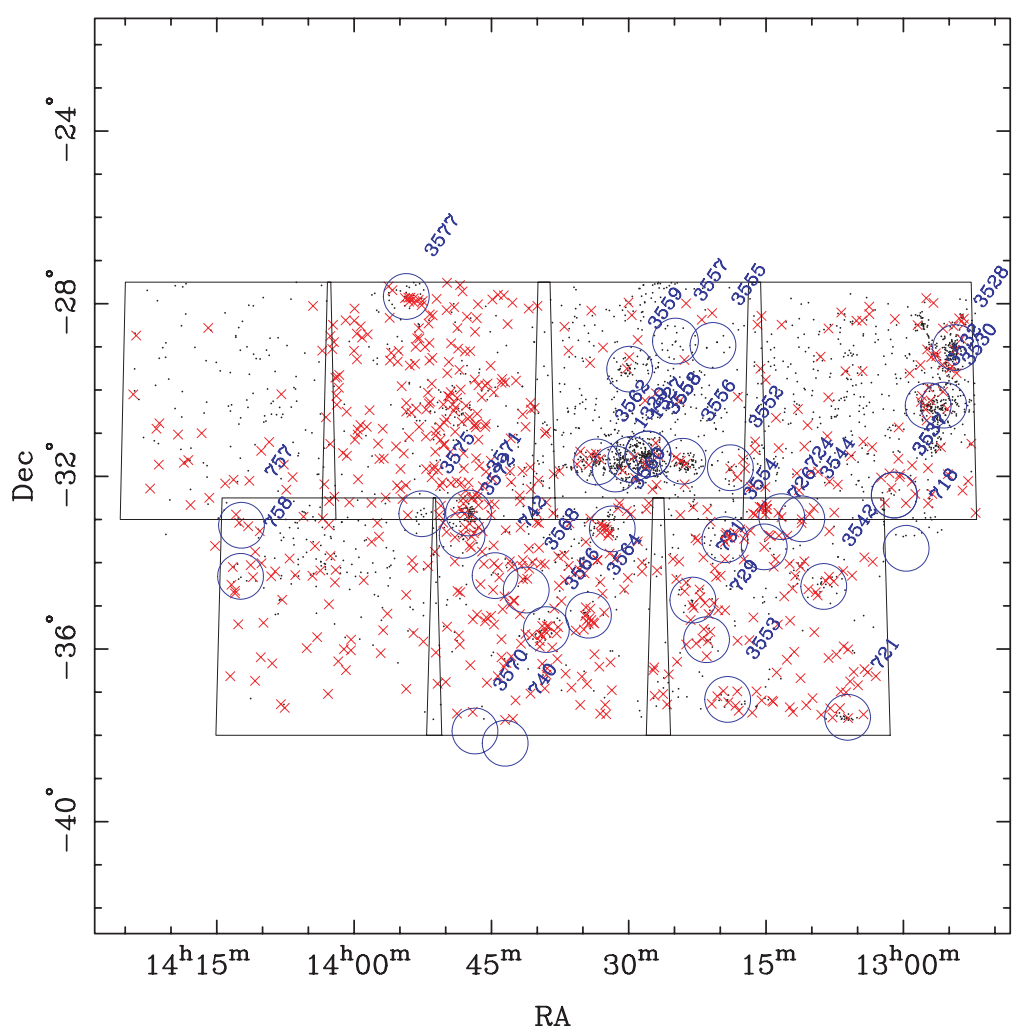

Figure 1 Sky distribution of galaxies with measured redshifts in the Shapley Region. The galaxies observed with FLAIR-II for this work are plotted as crosses and those previously published as dots. Known galaxy clusters with redshifts overlapping the Shapley range $\left(7500<c z<18500 \mathrm{~km} \mathrm{~s}^{-1}\right)$ are indicated by large labeled circles, and the 7 UKST fields by large overlapping squares. Note the concentration of previous measurements in the clusters.

galaxies on the sky. We also show galaxies with previously published redshifts and the known Abell clusters.

\subsection{Observed Galaxy Sample and Completeness}

For the purpose of our analysis below we wished to compare our sample of observed galaxies to the total magnitude-limited galaxy distribution in our survey region. We chose to use the new SuperCOSMOS sky surveys (Hambly et al. 2001a, 2001b, 2001c) to construct the parent galaxy catalogue for the region. This choice was motivated by the availabililty of full image data online from the SuperCOSMOS survey as well as some evidence that the MAMA photometry values used for the final two fields (384 and 446) were different from the others. We therefore quote and analyse the SuperCOSMOS R (the ' $R_{1}$ ' value from the UKST survey plates) magnitudes of all galaxies in this paper. In this system the sample does not have a sharp magnitude limit, as shown in Fig. 2. We compared the magnitude distributions of the objects measured in the seven fields: two-sample K-S tests showed that they were all consistent with the same distribution (mean $R \approx 15.8$ ) except for the two final fields -384 and 446 - which were significantly fainter (mean $R \approx 16.4$ ).

We next used the SuperCOSMOS survey to define a parent galaxy sample over the whole region covered by our seven observed fields. Allowing for the boundaries of the survey fields and a circular region 1 degree in diameter we excluded around the bright star HD 123139 (at $14^{\mathrm{h}} 06^{\mathrm{m}} 41^{\mathrm{s}} .0,-36^{\circ} 22^{\prime} 12^{\prime \prime}, \mathrm{J} 2000$ ), the total survey region has an area of 182 square degrees. As in D99, we also defined a restricted inter-cluster region by excluding regions 1 degree in diameter around any rich galaxy clusters in the Shapley velocity range, having an area of 159 square degrees. The excised cluster regions are shown in Fig. 1.

We used the parent galaxy sample to determine the completeness of our survey as a function of limiting magnitude. This is shown in Table 3 for both the full region and the inter-cluster region. As in D99, we have also compiled a larger list of galaxy measurements in the survey region based on published data (NED, searched 2003 Aug 5) and other new observations by our group (H. Quintana et al., in preparation). The table shows that the completeness is highest for the brighter magnitude limits, peaking at 28 per cent for the whole sample at $m_{R}<16$.

\section{Discussion}

We have obtained a total of new 710 galaxy radial velocities from all of our FLAIR-II observing runs. This represents a substantial improvement in the available 

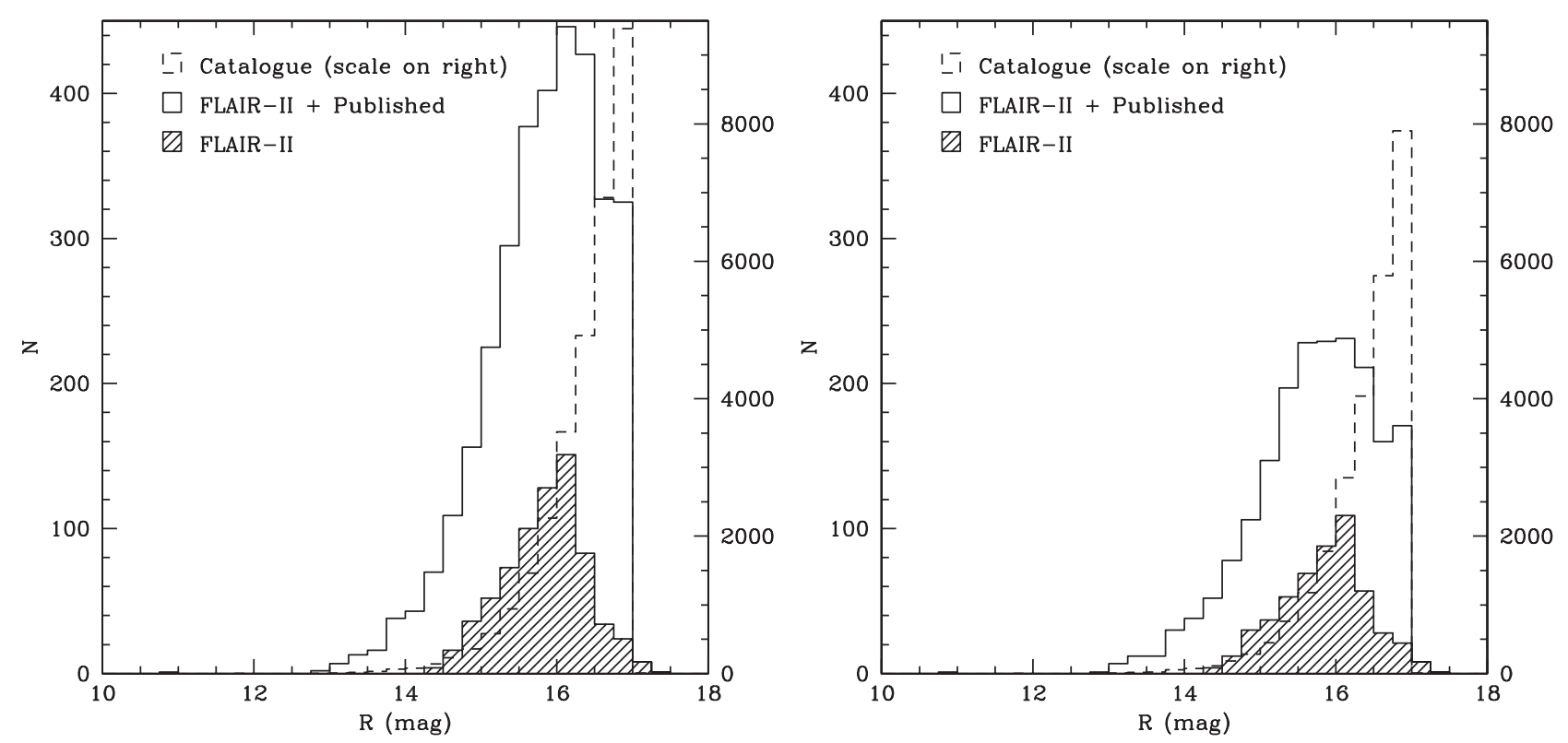

Figure 2 Magnitude distributions of observed and catalogued galaxies in the Shapley region. Left: full samples; Right: inter-cluster region only. Note that in each case the catalogue histogram is on a different scale, shown on the right.

Table 3. Galaxies Observed

\begin{tabular}{lcrll}
\hline Field & R Mag limit & Catalogue & FLAIR-II & FLAIR-II + Published \\
\hline Full & 16.0 & 6193 & $409(7 \%)$ & $1754(28 \%)$ \\
Full & 16.5 & 14635 & $643(4 \%)$ & $2627(18 \%)[10 \%]$ \\
Full & 17.0 & 30949 & $701(2 \%)$ & $3279(11 \%)[4.0 \%]$ \\
Full RA < 14:50 & 16.0 & 5309 & $397(7 \%)$ & $1630(31 \%)$ \\
Full RA < 14:50 & 16.5 & 12256 & $610(5 \%)$ & $2458(20 \%)$ \\
Full RA < 14:50 & 17.0 & 25484 & $658(3 \%)$ & $3087(12 \%)$ \\
Inter-cluster & 16.0 & 4930 & $293(6 \%)$ & $1138(23 \%)$ \\
Inter-cluster & 16.5 & 11819 & $459(4 \%)$ & $1580(13 \%)[6.4 \%]$ \\
Inter-cluster & 17.0 & 25511 & $508(2 \%)$ & $1911(7 \%)[2.4 \%]$ \\
\hline
\end{tabular}

Notes: the completeness of each sample compared to the catalogue is given in parentheses (integral) and brackets [differential].

velocity catalogue in the SSC, particularly for the previously neglected inter-cluster regions. In this section we use our sample to analyse the extent of the SSC and the galaxy overdensity it represents.

\subsection{Extent of the SSC}

Figure 3 shows the combined resulting distribution of galaxies towards the SSC as cone diagrams in right ascension and declination for both available literature redshifts and our new combined sample. The importance of the SSC in this region of the sky is demonstrated by the fact that $65 \%$ of galaxies in our new combined sample belong to the SSC with velocities in the range $8000-18500 \mathrm{~km} \mathrm{~s}^{-1}$. In both plots the new data are indicated by larger squares to emphasise their impact. It can be seen that by probing large regions of the SSC away from the rich Abell clusters, we have revealed significant additional structures which make complex links with the main cluster locations.
Figure 4 shows the histogram of 2030 galaxy redshifts in the direction of the SSC with all currently available published velocities (including our combined sample) in the range $0 \mathrm{~km} \mathrm{~s}^{-1} \leq V \leq 40000 \mathrm{~km} \mathrm{~s}^{-1}$ with a step size of $500 \mathrm{~km} \mathrm{~s}^{-1}$. The new velocities are also shown as the hatched region.

In agreement with previous works, we note the presence of a prominent foreground wall of galaxies (the Hydra-Centaurus region) at $\bar{V}=4000 \mathrm{~km} \mathrm{~s}^{-1}$. This distribution can be related to the nearby cluster A3627 associated with the 'Great Attractor' (Kraan-Korteweg et al. 1996). Moreover, our newly measured galaxies tend to establish a link between the Hydra-Centaurus region and the SSC, especially at the high declination end of the cone. A similar structure was suggested by Tully et al. (1992).

Concerning the main SSC, our new data revise our knowledge of its large-scale structure by measuring a large number of galaxies away from the rich Abell clusters previously studied. The majority $(65 \%)$ of the galaxies we 
observed were found to be part of the SSC, so our principal result is to show that the SSC is bigger and more complex than previously thought. Looking at the cone diagram in Right Ascension (Fig. 3), we can see that the SSC is separated into two main velocity components as previously suggested (D99). The nearer concentration at about $\bar{V}=11240 \mathrm{~km} \mathrm{~s}^{-1}$ is located to the East of the main SSC region at about $\bar{V}=14850 \mathrm{~km} \mathrm{~s}^{-1}$. However clumps of objects seem to link these two main structures which were not observed before. Care must be taken in the interpretation of the cone plots because of the finger of God effect evident in the main SSC concentrations due to the internal individual cluster velocity dispersions which can lead the eye to spurious links.

A large concentration of objects at about $\mathrm{RA}=12^{\mathrm{h}} 54^{\mathrm{m}}$ and around $\bar{V}=16000-17000 \mathrm{~km} \mathrm{~s}^{-1}$ (the 'A 3558' complex; see Bardelli et al. 2000) is connected to the main structure by a broad bridge of galaxies. It can also be seen from the declination cone diagram (Fig. 3, lower panel) that the Southern part of the SSC consists of two large sheets of galaxies, of which the previously measured Abell Clusters represent the peaks of maximum density. In particular, the
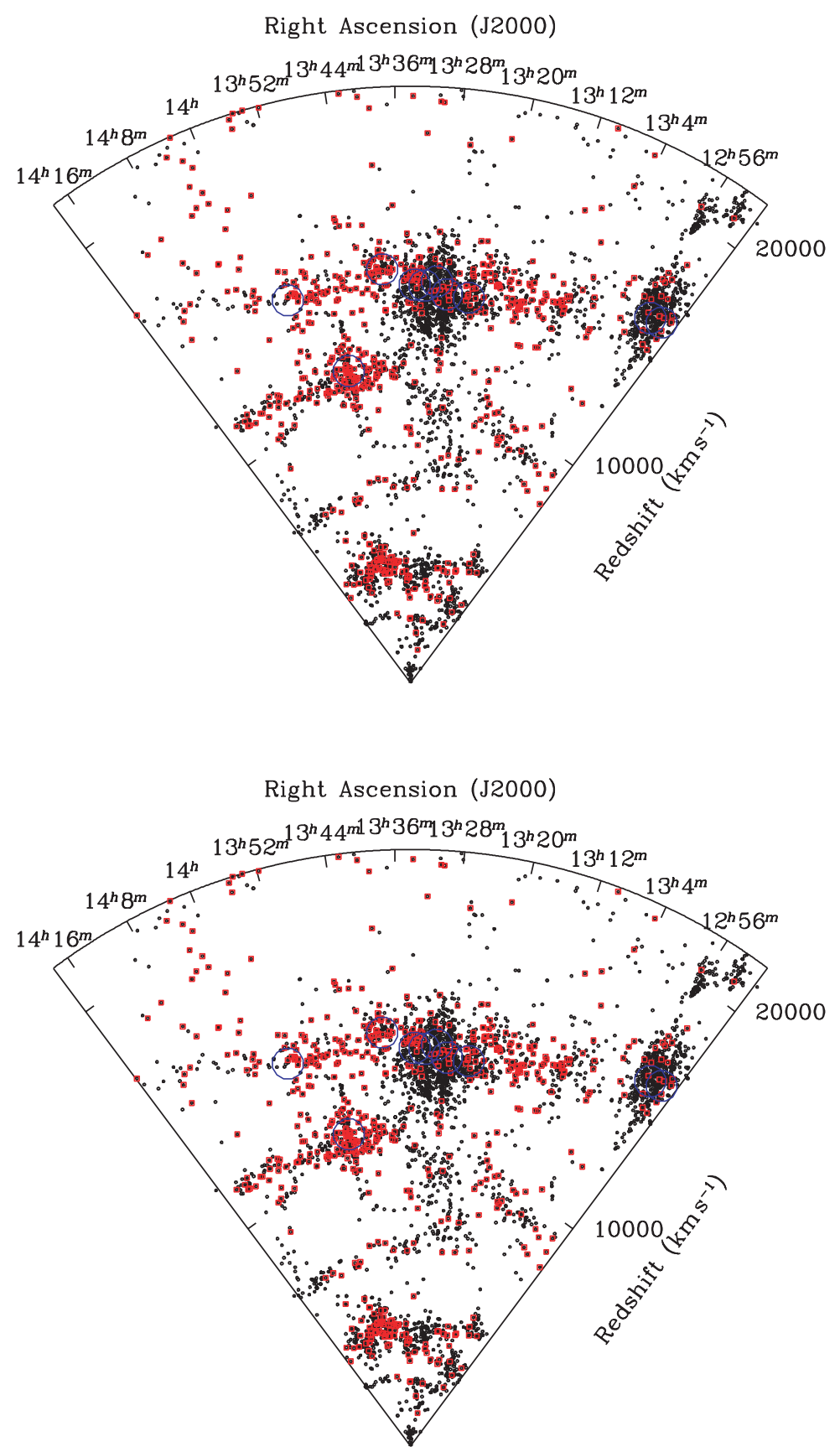

Figure 3 Cone velocity diagram in right ascension from $\mathrm{RA}=12^{\mathrm{h}} 15^{\mathrm{m}}$ to $\mathrm{RA}=14^{\mathrm{h}} 30^{\mathrm{m}}$ (upper panel), and declination from $-26^{\circ}$ to $-38^{\circ}$ (lower panel) for the measured galaxies in the Shapley Supercluster. Previously published galaxies are plotted as points; the combined new measurements from our project are plotted as squares. Also shown as large circles are the locations of the richest galaxy clusters in this region. 
more distant sheet, at $\bar{V}=15000 \mathrm{~km} \mathrm{~s}^{-1}$, is present right across the observed region from $-27^{\circ}$ to $-38^{\circ}$, so the true extent of this wall is currently unknown. The Southern part of this wall may be an extension of the plane of galaxies defined by Bardelli et al. (2000), although it has the same offset of $-5 \mathrm{~h}^{-1} \mathrm{Mpc}$ as our earlier D99 sample when analysed by Bardelli et al. in their Figure 4.

These new observations mean that we must re-appraise the conclusions of previous papers about the overall shape of the SSC. Based on the previously available velocity information on the main clusters, it was thought that the

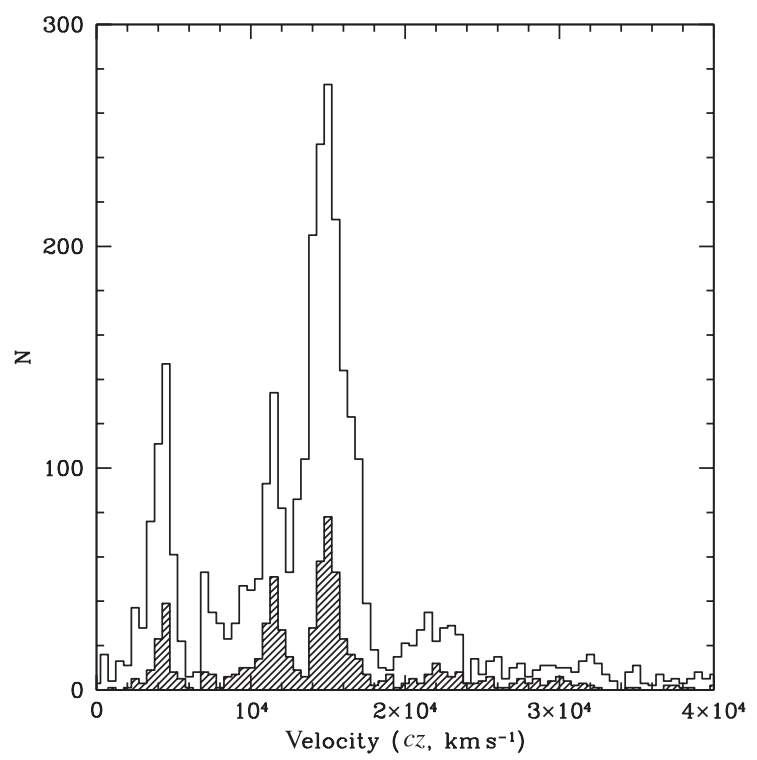

Figure 4 The redshift distribution of galaxies in the SSC survey region. The area of sky is $182 \mathrm{deg}^{2}$. The shaded histogram shows the 710 new measurements we present here and the upper histogram shows those combined with all know published redshifts, a total of 4215 galaxies. The bin size is $500 \mathrm{~km} \mathrm{~s}^{-1}$.

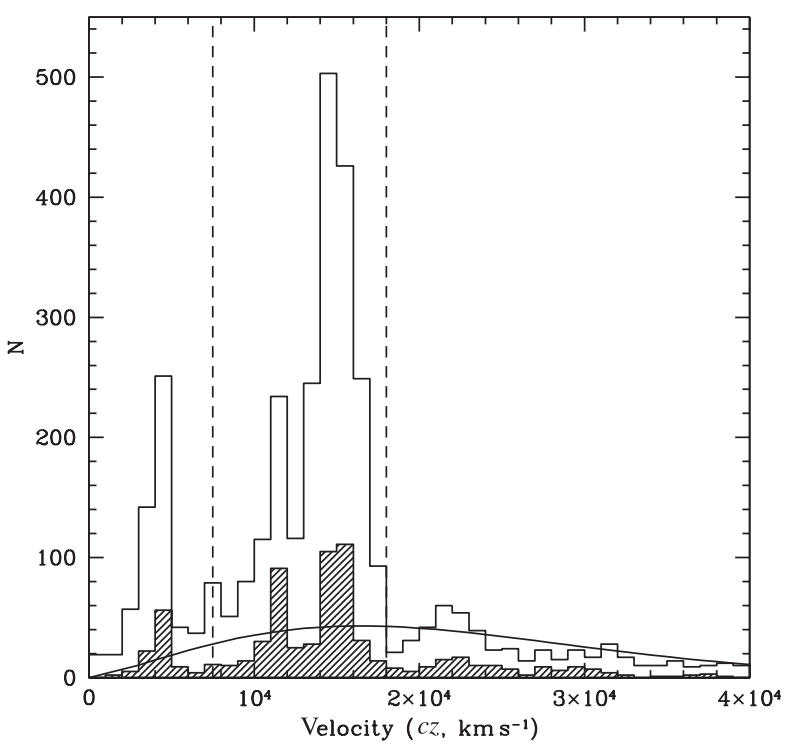

SSC was highly elongated and either inclined towards us or rotating. We now reveal that the SSC extends as far as our measurements do to the South via a broad interconnected wall so we find it is not elongated or flattened. The actual situation so far revealed is more complex, being composed of the known Abell clusters embedded in at least two sheets of galaxies of much larger extent.

\subsection{Galaxy Overdensity}

In D99 we estimated the galaxy overdensity in an intercluster region of area $44 \mathrm{deg}^{2}$ at the centre of the SSC region. Our new observations, combined with data from the literature, allow us to calculate the overdensity over a much larger region. We shall consider both our full survey region of $182 \mathrm{deg}^{2}$ and our new inter-cluster region of $151 \mathrm{deg}^{2}$. As we are most interested in galaxies at the distance of the SSC, the overdensity is best seen as the peaks in the redshift histograms.

In Fig. 5 we show the redshift distributions for both the full survey and inter-cluster regions. Also shown on both histograms are the expected distributions for a smooth, homogeneous galaxy distribution based on the number count data of Metcalfe et al. (1991). These were calculated allowing for the differential incompleteness of each sample as a function of $m_{R}$ as listed in the final column of Table 3 .

We calculated the galaxy overdensity as the ratio of the number of observed galaxies within the nominal velocity limits of the SSC complex (8000-18500 $\mathrm{km} \mathrm{s}^{-1}$ ) to the number expected from the Metcalfe counts within the same velocity limits, all with the magnitude limit of $m_{R}<17$. In the full region the overdensity was $5.0 \pm 0.1$ and in the restricted inter-cluster region it was $3.3 \pm 0.1$. This can be compared with our result in D99 where we found $2.0 \pm 0.2$ over an area of $44 \mathrm{deg}^{2}$. Our overdensity

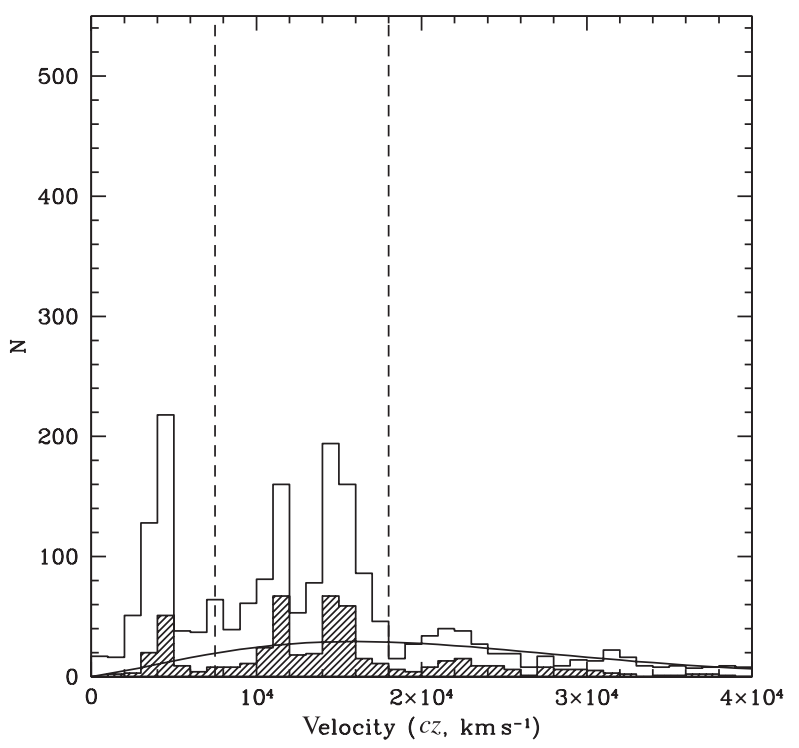

Figure 5 Redshift distributions of galaxies in the SSC region compared to that expected for a smooth, homogeneous galaxy distribution. The samples are drawn from (A) our full survey region and (B) the inter-cluster region. In each case the samples are limited to $m_{R}<17$. The expected distributions (Metcalfe et al. 1991) are scaled to the bin size, area and completeness of our samples. The dashed vertical lines indicate the nominal redshift range of the SSC, $7500-18000 \mathrm{~km} \mathrm{~s}^{-1}$. 
values are lower than those found by Bardelli et al. (2000) in a study of the central part of the SSC. They report an overdensity of $3.9 \pm 0.4$ for their inter-cluster sample and $11.3 \pm 0.4$ for their total sample, on scales of $10 \mathrm{~h}^{-1} \mathrm{Mpc}$. Our data probe the distribution on much larger scales of around $10-15$ degrees, corresponding to $26-40 \mathrm{~h}^{-1} \mathrm{Mpc}$. We have confirmed that earlier detection of a very significant galaxy overdensity in the inter-cluster space of the SSC region. In terms of galaxy numbers, the inter-cluster galaxies make up 46 per cent of the 2144 galaxies in the SSC velocity range, so assuming a similar mass function they contribute a similar amount of mass to the cluster galaxies which were the focus of most previous work on the SSC.

\section{Conclusion}

Our new observations of galaxies towards the SSC have, by surveying a large contiguous region including areas away from known clusters, revealed substantial new, large, inter-connected structures. The original SSC region has now been shown to form part of a much larger structure extending uniformly in at least two sheets over the whole surveyed region, principally to the South of the SSC core. We have detected an additional 426 members of the SSC in our combined (D99 and this paper) survey area, increasing very significantly the number of known SSC galaxies. Our new data suggest that the SSC is at least 50 per cent more massive than previously thought with a significant part of the extra mass in the closer sub-region. The SSC must therefore have a more important effect on the Local Group than originally envisaged. We defer a detailed calculation of the size of this effect and a fuller analysis and interpretation of these new data to a subsequent paper (D. Proust et al., in preparation) where some additional data will also be included.

We now plan to investigate regions well away from the SSC centre between RA $12^{\mathrm{h}} 30^{\mathrm{m}}$ and $14^{\mathrm{h}} 30^{\mathrm{m}}$ and declination from $-23^{\circ}$ to $-42^{\circ}$ in order to: (i) define the complete topology of the SSC; (ii) analyse the individual Abell clusters contained in the SSC; and (iii) determine more precisely its gravitational effect on the Local Group. We have already obtained the necessary extended catalogue of galaxies for this larger region from additional red ESO/SRC SSC plates scanned by the MAMA machine. The new $6 \mathrm{dF}$ replacement for FLAIR-II at the UKST with its automated robotic fibre positioner and 150 fibres in a $6^{\circ}$ field will be an ideal facility with which to continue our study of the SSC.

\section{Accessory Materials}

A catalogue of the 710 galaxies measured in this work are presented in the Accessory Materials, available from the authors or from PASA.

\section{Acknowledgments}

We wish to thank the UKST and AAO staff, especially Paul Cass, Malcolm Hartley and Ken Russell.
DP acknowledges receipt of a France-Australie PICS in support of visits to Siding-Spring Observatory. We are particularly grateful to Mike Read (ROE) for guidance with the SuperCOSMOS survey data. We also thank Maureen Younger (University of Queensland) for help with the galaxy identifications.

This research was partially supported by the cooperative programme ECOS/CONICYT C96U04 and HC thanks the FONDAP Centre for Astrophysics and the Guggenheim Foundation for partial support.

\section{References}

Bardelli, S., Zucca, E., Zamorani, G., Moscardini, L., \& Scaramella, R. 2000, MNRAS, 312, 540

Bardelli, S., Zucca, E., \& Baldi, A. 2001, MNRAS, 320, 387

Dickey, J. M., Keller, D. T., Pennington, R., \& Salpeter, E. E. 1987, AJ, 93, 788

Drinkwater, M. J., Currie, M. J., Young, C. K., Hardy, E., \& Yearsley, J. M. 1996, MNRAS, 279, 595

Drinkwater, M. J., Proust, D., Parker, Q. A., Quintana, H., \& Slezak, E. 1999, PASA, 16, 113 (D99)

Guibert, J., \& Moreau, O. 1991, The Messenger, 64, 69

Hambly, N. C., et al. 2001a, MNRAS, 326, 1279

Hambly, N. C., Irwin, M. J., \& MacGillivray, H. T. 2001b, MNRAS, 326,1295

Hambly, N. C., Davenhall, A. C., Irwin, M. J., \& MacGillivray, H. T. 2001c, MNRAS, 326, 1315

Heydon-Dumbleton, N. H., Collins, C. A., \& MacGillivray, H. T. 1989, MNRAS, 238, 379

Kaldare, R., Colless, M., Raychaudhury, S., \& Peterson, B. A. 2003, MNRAS, 339, 652

Kraan-Korteweg, R. C., Woudt, P. A., Cayatte, V., Fairall, A. P., Balkowski, C., \& Henning, P. A. 1996, Nature, 379, 519

Kurtz, M. J., \& Mink, D. J. 1998, PASP, 110, 943

Metcalfe, N., Shanks, T., Fong, R., \& Jones, L. R. 1991, MNRAS, 249,498

Moreau, O. 1992, PhD thesis, Université Paris, 7

Parker, Q. A., \& Watson, F. G. 1995, in 35th Herstmonceux Conf., Wide Field Spectroscopy and the Distant Universe, eds. S. J. Maddox, \& A. Aragon-Salamanca (Singapore: World Scientific), 33

Parker, Q. A. 1997, in Proc. 2nd Conf. of the Working Group of IAU Commission 9 on Wide-Field Imaging, Wide-Field Spectroscopy, eds. E. Kontizas, M. Kontizas, D. H. Morgan, \& G. P. Vettolani (Dordrecht: Kluwer Academic Publishers), 25

Quintana, H., Ramirez, A., Melnick, J., Raychaudhury, S., \& Slezak, E. 1995, AJ, 110, 463

Quintana, H., Carrasco, E. R., \& Reisenegger, A. 2000, AJ, 120, 511

Raychaudhury, S. 1989, Nature, 342, 251

Reisenegger, A., Quintana, H., Carrasco, E. R., \& Maze, J. 2000, AJ, 120, 523

Scaramella, R., Baiesi-Pillastrini, G., Chincarini, G., Vettolani, G., \& Zamorani, G. 1989, Nature, 338, 562

Smoot, G., et al. 1992, ApJ, 396, L1

Tody, D. 1993, in ASP Conf. Ser. 52, Astronomical Data Analysis Software and Systems II, eds. R. J. Hanisch, R. J. V. Brissenden, \& J. Barnes (San Francisco: ASP), 173

Tully, R. B., Scaramella, R., Vettolani, G., \& Zamorani, G. 1992, ApJ, 388, 9

Watson, F. G., Parker, Q. A., Bogatu, G., Farrell, T. J., Hingley, B. E., \& Miziarski, S. 2000, in Optical and IR Telescope Instrumentation and Detectors, Proc. SPIE, Vol. 4008, eds. I. Masanori, \& A. F. Moorwood (Bellingham: SPIE), 123 